\title{
Narrativa
}

\section{Vedersi vivere: vedersi apparire.Umorismo e identità di genere in Suo marito, di Luigi Pirandello}

\section{Daniela Brogi}

\section{(2) OpenEdition}

\section{Journals}

\section{Edizione digitale}

URL: https://journals.openedition.org/narrativa/535

DOI: $10.4000 /$ narrativa.535

ISSN: 2804-1224

\section{Editore}

Presses universitaires de Paris Nanterre

\section{Edizione cartacea}

Data di pubblicazione: 1 décembre 2018

Paginazione: 69-80

ISBN: 978-2-84016-325-1

ISSN: $1166-3243$

Notizia bibliografica digitale

Daniela Brogi, «Vedersi vivere: vedersi apparire.Umorismo e identità di genere in Suo marito, di Luigi Pirandello», Narrativa [Online], 40 | 2018, online dal 01 novembre 2021, consultato il 08 décembre 2021. URL: http://journals.openedition.org/narrativa/535 ; DOl: https://doi.org/10.4000/narrativa.535

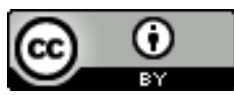

Narrativa est mise à disposition selon les termes de la Licence Creative Commons Attribution 4.0 International. 


\title{
Vedersi vivere: vedersi apparire. Umorismo e identità di genere in Suo marito, di Luigi Pirandello
}

\begin{abstract}
Riassunto
"Vedersi vivere": la formula chiave del saggio sull'Umorismo funziona, nell'intera opera pirandelliana, come dispositivo per rappresentare la scissione tra vita e forma. In Suo marito, il romanzo analizzato da Daniela Brogi, questa espressione diventa anche una strategia narrativa per costruire una storia, un sistema di personaggi e una forma di mascolinità che esiste in quanto si vede attraverso lo sguardo altrui.

\section{RÉSUMÉ}

"Se regarder vivre" : la formule clé de l'essai sur l'Humour fonctionne, dans toute l'œuvre de Luigi Pirandello, comme un dispositif qui permet de représenter la scission entre vie et forme. Dans Son mari, le roman analysé par Daniela Brogi, cette expression devient aussi une stratégie narrative qui permet de construire une histoire, un système de personnages et une forme de masculinité qui existe uniquement parce qu'elle est vue à travers le regard des autres.
\end{abstract}

\section{LA BRUTTA SCONOSCIUTA}

Cominceremo a parlare del quarto romanzo di Pirandello, Suo marito (Quattrini, Firenze, 1911) ${ }^{1}$, rileggendo il brano più noto del saggio sull'Umorismo (1908),

1. Alla prima edizione di Suo marito (1911) seguì una seconda edizione, postuma, del 1941 - in Tutti i romanzi, Mondadori - curata dal figlio Stefano e intitolata Giustino Roncella nato Boggiòlo. Si tratta di una versione nuova, rielaborata fino all'inizio del capitolo quinto. Le varianti si possono reperire nel volume primo di Tutti $i$ romanzi $i$ dei Meridiani, a cura di Giovanni Macchia con la collaborazione di Mario Costanzo, Milano, Mondadori, 1973. Per questo lavoro è stata seguita l'edizione 1911, così come si presenta, riproducendo il testo stabilito da M. Costanzo per I Meridiani, nella versione Oscar Mondadori curata da Laura Nay (Milano, 1994), a cui rimando usando la sigla 
vale a dire il punto in cui, definendo la scomposizione come principio estetico e formale più congeniale all'arte moderna, per chiarire la differenza tra l'“avvertimento del contrario" e il "sentimento del contrario" l'autore usa questa famosa immagine:

Vedo una vecchia signora, coi capelli ritinti, tutti unti non si sa di quale orribile manteca, e poi tutta goffamente imbellettata e parata d'abiti giovanili. Mi metto a ridere. Avverto che quella vecchia signora è il contrario di ciò che una vecchia rispettabile signora dovrebbe essere. Posso così, a prima giunta e superficialmente, arrestarmi a questa impressione comica. Il comico è appunto un avvertimento del contrario. Ma se ora interviene in me la riflessione, e mi suggerisce che quella vecchia signora non prova forse nessun piacere a pararsi così come un pappagallo, ma che forse ne soffre e lo fa soltanto perché pietosamente s'inganna che parata così, nascondendo così le rughe e la canizie, riesca a trattenere a sé l'amore del marito molto più giovane di lei, ecco che io non posso più riderne come prima, perché appunto la riflessione, lavorando in me, mi ha fatto andar oltre a quel primo avvertimento, o piuttosto, più addentro: da quel primo avvertimento del contrario mi ha fatto passare a questo sentimento del contrario. Ed è tutta qui la differenza tra il comico e l'umoristico ${ }^{2}$.

L'umorismo fu pubblicato nel 1908, tre anni prima di Suo marito, romanzo a cui Pirandello allude già in una lettera del 1904, usando anche per quest'opera l'aggettivo "umoristico". I due testi dunque appartengono, come raccontano le date, a una cronologia comune: di riflessioni, di scrittura, di temi. Nel brano appena riletto, alcuni motivi, in particolare, possono essere fissati come varchi speciali per una rilettura di Suo marito. In questa scena, infatti, Pirandello mette all'opera un repertorio di idee non privo tra l'altro, anche inconsapevolmente, di sfumature misogine. Il ritratto deformante, espressionista, della signora

Sm. Tra i lavori critici dedicati a Suo marito si segnalano Ricciardi Mario, Il posto di "Suo marito" nel romanzo pirandelliano, e Micocci Claudia, Silvia Roncella e/ o Giustino Boggiolo, contenuti in LauretTa Enzo (a cura di), Il "romanzo" di Pirandello, Palermo, Palumbo, 1976, pp. 109-124 e 125-141; Baldi Guido, Pirandello e il romanzo, Napoli, Liguori, 2006; Ganeri Margherita, Pirandello romanziere, Soveria Mannelli, Rubbettino, 2001.

2. Pirandello Luigi, L'umorismo e altri saggi, Ghidetti Enrico (a cura di), Milano, Giunti, 1998, p. 116.

3. Vedi lettera riportata alla fine dell'introduzione di Laura Nay all'Oscar Mondadori (p. LXXIV): "mi sono messo a scrivere intanto [...] un romanzo umoristico, assolutamente comico, intitolato Suo marito [...] riuscirà un libro divertentissimo": è una lettera autografa di Luigi Pirandello, datata "Girgenti, 17-IX-1904" indirizzata a tal "Dadone", contenuta nel catalogo della Libreria Antiquaria Pregliasco di Torino (n. 67, dicembre 1993, Lettere autografe e manoscritte). 
imbellettata fino al caricaturale per tenersi accanto un marito più giovane di lei ci parla anche di un sistema di rapporti di forza che diminuisce il femminile, ridicolizzandolo, chiudendolo in uno schema preciso di valori e disvalori schiacciato sul biologico (gioventù vs vecchiaia), secondo una "coscienza normale delle cose", come ci insegna a definirla proprio l'autore dell'Umorismo, per cui è impensabile che una donna agée possa "trattenere a sé l'amore" del coniuge. Al tempo stesso, questo sguardo fa famiglia con un immaginario primonovecentesco - tra donne brutte, sterili, femmine fatali divoranti e distruttive, isteriche, "arrampicatrici" sociali, ballerine goffe, madri arcigne continuamente abitato da metafore allarmate del femminile, metafore che ci parlano, in figura, di uno spettro ancora più inquietante, e dunque attaccato: quello della donna che lavora:

Il signor Ippolito non poteva soffrire le donne che portano gli occhiali, camminano come soldati, oggi impiegate alla posta, telegrafiste, telefoniste, e aspiranti all'elettorato e alla toga; domani, chi sa? alla deputazione e magari al comando dell'esercito ${ }^{4}$.

Del resto, anche il nostro romanzo, come vedremo, è pieno di "maschere" di donne invecchiate che agiscono, drammaturgicamente e visivamente, come riflessi umoristici di cui si adombra il racconto.

Nondimeno, definire e usare la figura introdotta nell'Umorismo ("Vedo una vecchia signora, coi capelli ritinti, tutti unti non si sa di quale orribile manteca, e poi tutta goffamente imbellettata e parata d'abiti giovanili ") considerandola semplicemente come un'immagine non basta, perché il testo fa qualcosa di più importante: reinventa e compone una piccola narrazione, teatralizzando il tema della scoperta dell'altro (dell'altra in questo caso) nella forma di un "avvertimento" in quanto percezione. E così lo spunto di una vecchia donna diventa il racconto di una "visione" scaturita da un incontro che si compie attraverso un'azione espressa da un presente generico ("vedo") e diretta su un soggetto anonimo ("una signora") che comincia a prendere vita narrativa funzionando come una specie di spettacolo che si offre allo sguardo dell'artefice del racconto. Tutto è indeterminato, spersonalizzato, come le situazioni che accadono nello scenario della vita trascorsa, per così dire, in pubblico: negli spazi e nei tempi passati a vedere gli altri e a farci vedere. Per certi versi si potrebbe perfino considerare questo testo come una sorta di riscrittura, in tutt'altra chiave, della situazione

4. Pirandello Luigi, Suo marito [1911], Milano, Oscar Mondadori, 1994, pp. 42-43. 
messa in scena dalla famosa poesia di Baudelaire $\grave{A}$ une passante. La persona appariscente (la "facciata", come avrebbe detto Goffman"), l'abbigliamento vistoso della signora grottescamente imbellettata compongono una situazione narrativa e visuale che per esistere ha bisogno di un set sociale: un ambiente dove si svolge una sorta di rappresentazione, di messa in scena di sé. L'immagine di quella signora vive attraverso uno scambio, una negoziazione che passa tra lo sguardo degli altri, e un'intenzione (definita più avanti nel testo) di apparire, ovvero di essere, qualcosa che non si è più, cioè attraente come una donna giovane. Questo è un primo, importante motivo che ci aiuterà a capire meglio il romanzo di cui ci occuperemo, fin dal suo titolo. Il romanzo che più di tutti, tra le opere pirandelliane, sa rappresentare il mondo della Belle Époque italiana, tra atmosfere mondane che fanno ripensare a Vanity Fair (1848) di Thackeray, e a Bel Ami (1885) di Maupassant, e persino a Il piacere (1889) di D'Annunzio. Suo marito, del resto, comincia proprio con una festa: il banchetto (è anche il nome del primo capitolo del romanzo) organizzato in occasione del debutto romano della nuova autrice Silvia Roncella. Vedersi vivere è anche vivere vedendosi agire sulla scena del mondo, dando valore e identità di genere a sé stessi attraverso il consenso sociale e l'esibizione di una comune appartenenza a un certo orizzonte di gusto e di distinzioni indispensabile per fare "bella figura":

Ama la musica la vostra signora?

- Credo - rispose Giustino, impacciato. - Non so bene... Ne ha sentita poca... là, a Taranto. Perché, si fa molta musica in casa della Marchesa?

- Talvolta sì, - disse Dora. - Viene il violoncellista Begler, il Milani, il Cordova, il Furlini, e s'improvvisa il quartetto...

- Eh già, - sospirò Giustino. - Un po’ di conoscenza della musica... di quella difficile... oggi è proprio necessaria... Wagner...

- No, Wagner, col quartetto! - esclamò Dora. - Tchaikowsky, Dvorak... e poi, si sa, Glazounov, Mahler, Raff.

- Eh già, - sospirò di nuovo Giustino. - Tante cose si dovrebbero sapere...

- Ma no! basta saperli pronunziare, caro Boggiolo! - disse Dora, ridendo.

- Non vi date pensiero. Se non dovessi guardarmi la professione, scriverei io un libro, che vorrei intitolare La Fiera o il Bazar della Sapienza... Proponetelo a

5. Goffman Erving, Rappresentazioni di genere [Gender Advertisements, 1979], Romeo Angelo (a cura di), postfazione di Codeluppi Vanni, Milano, Mimesis, 2015. 
vostra moglie, Boggiolo. Ve lo dico sul serio! Le darei io tutti i dati e i connotati e i documenti.

Una filza di questi nomi difficili... poi un po' di storia dell'arte... - basta leggere un trattatello qualunque - un po' d'ellenismo, anzi di pre-ellenismo, arte micenaica e via dicendo, - un po' di Nietzsche, un po' di Bergson, un po' di conferenze, e avvezzarsi a prendere il the, caro Boggiolo. Voi non ne prendete, e avete torto. Chi prende il the per la prima volta, comincia subito a capire tante $\operatorname{cose}^{6}$.

Il secondo motivo, invece, che dal saggio sull'Umorismo a Suo marito intesse i fili di una significativa ricorrenza, è che nel brano citato all'inizio, come accade pure in molte opere teatrali, Pirandello usa e inventa il femminile trasformandolo in una sorta di motto di spirito, vale a dire come immagine e gesto perturbanti, "osceni" nel senso pieno di situazione solitamente lasciata fuori dall'inquadratura, ma che invece ritorna, riprendendosi la scena attraverso parole, funzioni e ruoli fuori asse e fuori norma, che funzionano, complessivamente, come segni, significanti di una prospettiva umoristica. Il destino anomalo del protagonista di Suo marito, in effetti, è tutto costruito, messo all'opera, tenuto in equilibrio (per i primi quattro capitoli ${ }^{7}$ ) e poi fatto squilibrare, in senso tecnico, attraverso le azioni e le reazioni intorno alla figura opaca e straniante di sua moglie: Silvia Roncella. La vanità (di successo) e la vergogna (di successo) rispettivamente personificate dalle maschere di Giustino e Silvia sono i due poli attorni ai quali si muove la vicenda e il sistema di significati messi all'opera dal romanzo più mondano di Pirandello: Suo marito.

\section{LA TRAMA E IL TITOLO}

"Mi sono messo a scrivere intanto [...] un romanzo umoristico, assolutamente comico, intitolato Suo marito [...] riuscirà un libro divertentissimo", "manderò pure al Treves, spero in aprile, il romanzo Suo marito. Son partito dal marito di Grazia Deledda. Lo conosci? Che capolavoro, Ugo mio! Dico, il

6. Pirandello Luigi, Suo marito, cit., pp. 72-73.

7. La struttura di Suo marito è organizzata in sette capitoli, comprendenti altri sottocapitoli, che si succedono con questo ordine e titolo: I: Il banchetto (5 capp.); II: Scuola di grandezza (4 capp. ); III: Mistress Roncella two accouchements (4 capp. ); IV: Dopo il trionfo (4 capp. ); V: La crisalide e il bruco (4 capp. ); VI: Vola via (4 capp. ); VII: Lume spento (4 capp.).

8. Cfr. nota 3. 
marito di Grazia Deledda - intendiamoci...". Secondo quanto dice lo stesso autore in alcuni cenni epistolari, il marito a cui si ispira il romanzo è Palmiro Madesani, un funzionario pubblico che aveva sposato la scrittrice Grazia Deledda l'undici gennaio 1900, e che, dopo il trasferimento a Roma, aveva lasciato il proprio lavoro diventando agente a tempo pieno e per certi aspetti sapiente artefice della carriera artistica della moglie:

Perché veramente per Giustino Boggiolo il gigante non era il dramma composto da sua moglie; il gigante era il trionfo, di cui egli solamente si riconosceva l'autore. Ma sì! Se non ci fosse stato lui, se lui non avesse operato miracoli in tutti quei mesi di preparazione, ora difatti tanta gente sarebbe accorsa lì, alla stazione, a ossequiar la moglie, a felicitarla, ad augurarle il buon viaggio! ${ }^{10}$

Tutti capirono a chi si alludeva; Deledda si offese; l'editore Treves rifiutò di pubblicare il libro, che effettivamente non fu più ristampato, e, tra i romanzi pirandelliani, è rimasta l'opera meno letta - per certi aspetti più rimossa. Suo marito è la storia della conquista del successo di Silvia Roncella, giovane autrice giunta a Roma da Taranto, assieme al suo sposo, che grazie alle opere teatrali e alla trama di relazioni mondane intessute dal marito conquisterà fama e successo di pubblico. Ma, dopo una prima fase di timida inerzia (non priva di spunti di passività narcisistica) e di indiscussa accettazione del protagonismo del marito, capace di ignorare il disprezzo e la derisione pur di garantire la fortuna dell'opera della moglie, di cui si assume, in un certo senso, la paternità simbolica, Silvia comincerà a prendere distanza dal coniuge, fino a separarsene.

Riassumere la trama di Suo marito, che si svolge a partire dall'aprile 1908, è un'operazione semplice (se ci limitiamo ai nudi materiali ripresi) e complicata (se guardiamo alla loro rielaborazione creativa), perché il libro costruisce una storia intenzionalmente ambigua, come già fa capire il titolo, che adombra, dicendo ma senza dire del tutto, un clima di ostilità, un pensiero malevolo; e lo fa intanto che lavora dentro il modello di relazione narrativa e simbolica che più di tutte, nella modernità, ha reso visibile, riconoscibile e dunque significativo l'io in quanto io sociale, vale a dire l'istituto del matrimonio. Ad eccezione dei Quaderni di Serafino Gubbio operatore (1925), ciascuno di tutti gli altri romanzi pirandelliani, infatti, usa il matrimonio come scenario decisivo, ma Suo marito fa

9. Pirandello Luigi, Carteggi inediti (con Ojetti - Alberini - Orvieto - Novaro - De Gubernatis - De Filippo), Zappulda Muscarà Sarah (a cura di), Roma, Bulzoni, 1980, p. 28.

10. Pirandello Luigi, Suo marito, cit., p. 115. 
qualcosa di più sottile, perché trasforma il vincolo tra due coniugi in prospettiva testuale e umoristica primaria, dall'interno della quale reinventare e riguardare la relazione, estrovertendola, vale a dire non trattandola soltanto come un dramma intimo e privato. Rappresentando la vicenda attraverso una sorta di superocchio mondano che comincia a esistere, a guardare e a parlare, quasi come un personaggio fantasma, fin dalla vox populi che dà il titolo alla copertina, Suo marito sviluppa, in un certo senso prendendolo alla lettera - vale a dire tematizzandolo, tanto nel senso della scena sociale quanto nel senso del mondo teatrale in cui Silvia, realmente, conquista la celebrità - il rivoluzionario motivo pirandelliano dell'io come maschera e come sdoppiamento continuo: tra ciò che si è e ciò che si appare. "Vedersi vivere", la formula chiave del saggio sull'Umorismo (1908) che viene ripresa anche in Suo marito (1911), funziona infatti, nell'intera opera pirandelliana, come dispositivo compositivo e metaforico per indicare e rappresentare la scissione tra vita e forma. Ma proprio in Suo marito, più che mai, questa espressione diventa anche una strategia narrativa specifica per costruire una trama, una storia, un sistema di personaggi, un babitus sociale. Farsi ammirare, allora, è l'imperativo che funziona anche come modello di genere, come forma di mascolinità che esiste in quanto si vede, e si finge, attraverso lo sguardo altrui.

L'incipit, da questo punto di vista, costruisce immediatamente un ironico incrocio di sguardi e di rispecchiamenti tra maschile e femminile, messi in scena, entrambi, come identità artefatte, che si guardano a vicenda, dentro una situazione umoristica, e illuminandosi reciprocamente di ambiguità:

Attilio Raceni, da quattro anni direttore della rassegna femminile (non femminista) Le Muse si svegliò tardi, quella mattina, e di malumore.

Sotto gli occhi delle innumerevoli giovani scrittrici italiane, poetesse, novellatrici, romanzatrici (qualcuna anche drammaturga), che lo guardavano dalle fotografie disposte in vari gruppi alle pareti, tutte col volto composto a un'aria particolare di grazia vispa o patetica, scese dal letto - oh Dio, in camicia da notte naturalmente, ma lunga, lunga per fortuna fino alla noce del piede. Infilate le pantofole, andò a spalancar la finestra ${ }^{11}$.

Svegliarsi tardi, di malumore, in una lunga camicia da notte: tutti i tratti che definiscono il primo personaggio in scena, in Suo marito, sono segni di un babitus più spesso usato per costruire il comportamento femminile che maschile. E questo comico effetto di avvertimento del contrario continua, mentre assistiamo, come nella scena di un film muto, al gioco di sguardi tra Attilio e i simulacri delle

\section{Ibid., p. 3.}


tante donne che lo spiano dalle pareti. Svegliarsi, tornare alla vita, e cominciare a essere guardato da qualcun altro compongono un'unica situazione.

Ispirandosi alla vicenda del successo della scrittrice Grazia Deledda, Suo marito è un romanzo costruito, umoristicamente, intorno a tre motivi principali: alla celebrità letteraria; al tema e all'ideologia della creatività femminile come forma di continua scissione tra un destino privato e un destino femminile; e alle sorti di un matrimonio, messo alla prova, scompensato, da un anomalo rovesciamento di rapporti di forza per cui è la moglie a possedere la fama - e con essa il capitale simbolico, economico, e potremmo aggiungere anche narrativo, della coppia, cognome compreso. Ora, proprio questa terza prospettiva testuale della relazione coniugale funziona come scheletro del racconto, attorno al quale prende forma e figura un organismo del tutto straniante: una specie di corpo estraneo rispetto all'ossatura di partenza, che interpella il lettore - altre volte lo spettatore - inducendo ora distanza ora senso del ridicolo e del comico, ora sentimento del contrario. Se la prima parola del titolo Suo marito richiama subito l'idea di un possesso, la seconda parola, infatti, introduce immediatamente la prospettiva particolare e sfalsata da cui si riconsidera quel possesso. Al contrario di quanto direbbe l'ordine delle cose cristallizzato dalle etimologie, i due coniugi, infatti, possiedono matrimonio (: possesso della madre) e patrimonio (: possesso del padre) in maniera rovesciata e umoristica rispetto agli assetti comuni, perché Silvia, in realtà, possiede il patrimonio (garantito dai proventi delle sue opere) e Giustino possiede il matrimonio - tant'è vero che per stigmatizzare, deridendola, l'inferiorità di Giustino rispetto alla moglie, i suoi colleghi gli faranno stampare dei biglietti da visita con il cognome di Silvia: Giustino Roncella ( $\mathrm{Sm}$, p. 98); allo stesso modo per cui il signor Crowell, il corrispondente dall'Italia di The Nation - New York giunto a intervistare Silvia la definirà "Mistress" (Sm, pp. 74). Suo marito, in un certo senso, non è, come abbiamo detto sin qui, la storia della conquista del successo di Silvia Roncella, bensì, secondo un progetto umoristico di scomposizione prospettica, la storia della conquista e della perdita del successo di "suo marito": Giustino Boggiolo.

\section{UMORISMO E MASCOLINITÀ}

"Quanto aveva sofferto durante quel banchetto, esposta lì, come a una fiera! S'era veduta quale un automa mal congegnato, a cui si fosse sforzata la carica" ${ }^{2}$.

12. Ibid., p. 59. 
Vanità e vergogna sono i due sentimenti che più contraddistinguono, e dividono, le reazioni di Giustino e di Silvia dinanzi al successo. In questo senso, il destino di Silvia per certi aspetti ci parla, come faceva in quegli stessi anni un romanzo apprezzato da Pirandello, Una donna (1906), di Sibilla Aleramo, di un motivo importante e spesso ripreso dalla narrativa modernista europea, vale a dire il destino dell'artista donna, delle scissioni e delle ambivalenze legate alla doppia gestazione di un figlio e di un'opera letteraria. Un motivo che, anche in questo caso, conosce un inconsueto trattamento umoristico:

- Sissignora, sissignora... proprio così, - confermò il Boggiolo. [...] Non ci voleva, giusto in questo momento, il guajo della gravidanza...

- Vedete? - esclamò Dora. - E la vostra signora, chissà quanto soffrirà poi a staccarsi dal bambino!

- Ma! - fece il Boggiolo, - dovendo lavorare...

- È molto triste! - sospirò la Barmis. - Un figliuolo!... Dev'essere terribile vedersi, sentirsi madre! Io morrei di gioja e di spavento! Dio Dio Dio non mi ci fate pensare ${ }^{13}$.

Ma, a rendere ancora più interessante Suo marito, è il trattamento plastico dei personaggi, che nel corso del racconto mutano atteggiamenti, e funzioni, sfuggendo a forme univoche di identificazione o pure a modelli chiusi di straniamento: l'umorismo agisce come procedimento dinamico, impulso al rovesciamento continuo dei ruoli e delle maschere. Così, attraverso il racconto dei successi di Silvia, come dei suoi conflitti coniugali, in realtà il romanzo di certo aggredì la coppia di Grazia Deledda e suo marito, ma mette in scena anche risonanze significative con la biografia pirandelliana. Non per nulla, Silvia, autrice di un romanzo proclamato come un "capolavoro d'umorismo" 14 , proprio come Pirandello, progressivamente si dedicherà al teatro, e la narrazione le attribuisce titoli di opere pirandelliane - La nuova colonia, o Se non cosi, per esempio.

Ma anche Giustino, da parte sua, è un personaggio meno semplice di quanto non possa apparire, perché il suo mimetismo goffo da parvenu, la sua incapacità di saper scegliere gli abiti giusti presentandosi in marsina a un Lunch ( $\mathrm{Sm}$, p. 29), la sua fame di successo, sono senza dubbio oggetto di uno sguardo ostile, subito pronto a cogliere i lati ridicoli del personaggio, tanto dentro la storia (attraverso

13. Ibid., p. 69-70.

14. Ibid., p. 27. 
l'occhio degli altri, e persino, da un certo punto in là, di Silvia), quanto all'esterno; nondimeno, Giustino garantisce al racconto, attraverso la sua sfacciata imprenditorialità, anche una sua verità romanzesca, sia per la capacità di rappresentare il campo letterario primonovecentesco:

- Eppoi studiai la legge su la proprietà letteraria, sicuro! e anche il trattato di Berna sui diritti d'autore... Eh, la letteratura è un campo, signora mia, da contrastare allo sfruttamento sfacciato della stampa e degli editori. Ne hanno fatte tante anche a me, nei primi giorni! Contrattavo così, tentoni, si sa... Ma poi vedendo che le cose andavano... Silvia si spaventava dei patti che facevo; nel vedere poi accettati i prezzi, quando le mostravo il denaro guadagnato, rimaneva soddisfatta... eh sfido! Però, sa, posso dire d'averlo guadagnato io, il denaro, perché ella dai suoi lavori non avrebbe saputo cavare mai nulla ${ }^{15}$.

sia per il modo in cui la trama, costruendo un gioco continuo di scambi e di simmetrie tra Silvia e Giustino, produce, nel tempo, un progressivo spostamento dall'avvertimento del contrario al "sentimento" del contrario anche nei confronti di Giustino. E così, la narrazione del doppio parto della coppia Roncella-Boggiolo si trasforma nella portentosa sequenza di una tripla rappresentazione (il parto di Silvia, fuori campo; l'allestimento della sua opera, sullo sfondo; la reazione di Giustino, in primo piano) dentro la rappresentazione e gli effetti di umorismo composti dalla scrittura. Ecco che il vero travaglio riconosciuto, reso visibile dalla narrazione, diventa, paradossalmente, quello di Giustino:

Era gonfio d'orgoglio, ora, pensando che già era uno splendido e magnifico spettacolo per sé stesso quel teatro così pieno, e che si doveva a lui: opera sua, frutto del suo costante, indefesso lavoro, la considerazione di cui godeva la moglie, la fama di lei. L'autore, il vero autore di tutto, era lui. [...] Man mano che la rappresentazione procedeva, una violenza strana, un fascino teneva e legava lì Giustino, sgomento, come al cospetto d'un fenomeno mostruoso: il dramma che sua moglie aveva scritto, ch'egli sapeva a memoria parola per parola, e che finora aveva quasi covato, ecco, si staccava da lui, si staccava da tutti, s'inalzava, s'inalzava come un pallone di carta ch'egli avesse diligentemente portato lì, in quella sera di festa, tra la folla, e che avesse a lungo e con cura trepidante sorretto su le fiamme da lui stesso suscitate perché si gonfiasse, a cui ora infine egli avesse acceso lo stoppaccio; si staccava da lui, si liberava palpitante e luminoso, si inalzava, si inalzava nel cielo, traendosi seco tutta la sua anima pericolante e quasi tirandogli le viscere, il cuore, il respiro, nell'attesa angosciosa che da un istante all'altro un buffo d'aria, una scossa di vento, non

15. Ibid., p. 69. 
lo abbattesse da un lato, ed esso non s'incendiasse, non fosse divorato lì nell'alto dallo stesso fuoco ch'egli vi aveva acceso. [...] Il Raceni lo aveva raccolto tra le braccia, sul petto, singhiozzante e lo sorreggeva, mentre quattro, cinque volte gli attori si presentavano alla ribalta, a quell'incendio là... Egli singhiozzava, rideva e singhiozzava e tremava tutto di gioja. Dalle braccia del Raceni cadde tra quelle della Carmi, e poi del Revelli, e poi del Crimi che gli stampò su le labbra, su la punta del naso e sulla guancia i colori della truccatura, perché in un impeto di commozione egli volle baciarlo a ogni costo, a ogni costo, non ostante che quegli, sapendo il guaio che ne sarebbe venuto, si schermisse. E col volto così impiastricciato, seguitò a cadere tra le braccia dei giornalisti e di tutti i conoscenti accorsi sul palcoscenico a congratularsi; non sapeva far altro; era così esausto, spossato, sfinito, che solo in quell'abbandono trovava sollievo; e ormai s'abbandonava a tutti, quasi meccanicamente; si sarebbe abbandonato anche tra le braccia dei pompieri di guardia, dei macchinisti, dei servi di scena, se finalmente a distoglierlo da quel gesto comico e compassionevole, a scuoterlo con una forte scrollatina di braccia non fosse sopravvenutala Barmis, che lo guidò nel camerino della Carmi per fargli ripulir la faccia. Il Raceni era scappato a casa a prender notizie della moglie ${ }^{16}$.

Il personaggio di Giustino è un capolavoro di umorismo. Gli effetti di rovesciamento prospettico di questa pagina fanno parte di una strategia complessiva per cui l'umorismo è, per l'appunto, conquista di uno sguardo tridimensionale nel tempo: della storia come del racconto. I capitoli finali di Suo marito, quelli in cui Giustino, costretto a prendere atto della conquista di autonomia e l'allontanamento di Silvia, si tirerà fuori dallo spazio mondano di Roma, per ritirarsi dentro lo spazio della madre, dove più avanti si consumerà la morte del bambino di Silvia e Giustino, in significativa simmetria con il trionfo definitivo di Silvia, sono pagine melodrammatiche, indubbiamente, ma che piegano il melodramma al progetto di un'empatia che, secondo la poetica pirandelliana, non è limite ma condizione del "sentimento del contrario".

Suo marito in questa prospettiva è il romanzo più mondano di Pirandello non solo perché dedicato alla società letteraria della Belle époque romana raccontata nel suo complesso; ma pure perché tratta l'ideale dell'identità maschile come livello testuale significativo per mettere in romanzo il mondo, e rappresentarlo umoristicamente - come già dicono il titolo, così spiazzante, del libro e la scelta stessa, straniante, di parlare della vanità di successo attraverso il riflesso del maschile anziché del femminile. Giustino Boggiolo, il marito, e Maurizio Gueli, l'amante di Silvia, che dopo una fuga d'amore tornerà dalla compagna, sono

16. Ibid., p. 104-108. 
modelli significativi di un io maschile che per un verso agisce in una specie di città delle donne caricaturale: abitata dai fantasmi più ricorrenti del femminile partoriti dalla fantasia maschile che si sente minacciata (la letterata ammaliante, la donna sterile, la moglie possessiva e dominatrice, l'attrice egocentrica e vendicativa, la vecchia madre vittimistica e la giovane madre anaffettiva); per l'altro verso, l'io maschile si forma continuamente sotto lo sguardo e i giudizi degli altri. Il comportamento del marito e dell'amante di Silvia, la loro verità romanzesca funziona anche, man mano che si vedono vivere e vivono vedendosi, come una smentita continua a una richiesta di superomismo e di "virilità" che tanto Giustino che Maurizio, per arrivismo goffo, o per narcisismo imploso, deludono continuamente, infrangendo e strappando il cielo di carta delle apparenze sociali, fino a soccombere.

Attraverso questo sistema di rovesciamenti e di scambi che fanno esplodere la grammatica convenzionale dei generi (maschile versus femminile $=$ forza vs debolezza $=$ attivo $v$ p passivo $=$ successo pubblico $v s$ dismissione creativa), Suo marito, raccontandoci il destino così anomalo, non conforme, del protagonista mette in scena, facendolo vivere e facendocelo vedere scenicamente e melodrammaticamente, anche un sistema più fluido delle identità di genere, nel senso che il romanzo rappresenta un mondo che di lì a poco la cultura fascista avrebbe appiattito e reso uniforme, imponendo, anche oltre il ventennio, un'idea - un'ideologia - di mascolinità da cui il Novecento italiano (le sue opere, e spesso pure le sue sistemazioni critiche) è stato molte volte ingombrato.

Daniela BROGI

Università per Stranieri di Siena 